\title{
Mathematical modeling of processes of heat and mass transfer during drying of wood biomass
}

\author{
Natalya Ivanova ${ }^{l, *}$, and Elena Bulba ${ }^{1}$ \\ ${ }^{1}$ National Research Tomsk Polytechnic University, 634050 Tomsk, Russia
}

\begin{abstract}
For the first time, a mathematical model for the drying of woody biomass during conductive heating with localization of the evaporation front has been formulated. The processes of moisture removal during the filtration of steam through the porous structure of the material at an ambient temperature of $\mathrm{Te}=373 \mathrm{~K}$ were considered. Humidity was varied (in the range from $6 \%$ to $40 \%)$ and dimensions of wood blanks ( $\mathrm{Rd}=0.0035-0.035$ $\mathrm{m})$. Based on the results of numerical simulation, the conditions and characteristics (evaporation rate Wisp, drying time $\tau$ dry) of the process of moisture removal from wood biomass are determined. The mathematical model allows to calculate the drying time, as well as the mass evaporation rate for different sizes of wood sample, humidity and temperature conditions.
\end{abstract}

\section{Introduction}

The use of wood waste in the energy sector is ecologically and energy-efficient [1]. The latter is because that the wood biomass is carbon-neutral, the sulfur and nitrogen content in the wood is lower than the traditional solid (coal) [2], liquid (oil, fuel oil) and gaseous (natural gas) energy carriers $[3,4]$.

Progress in wood drying technologies is possible by studying the regularities of heat and mass transfer processes [5]. Many of physical and structural factors that find the result of intense thermal effects on wood make it impossible to study the mechanism of drying experimentally. For this reason, the most acceptable at this stage in the development of the theory and practice of the drying of moist wood biomass is the use of the apparatus of mathematical modeling.

But it is worth noting that the developed mathematical models of drying are based, as a rule, on substantially simplifying the procedure for modeling the assumptions [6]. For example, in [7], a one-dimensional model of heat and mass transfer of the processes occurring when drying wet wood biomass is considered. Application of the statement [7] is possible only when studying the processes of moisture removal in bodies possessing axial symmetry (a cylinder, a ball). In this case, it is impossible to consider the real configuration of the body under conditions of uneven surface heating.

In [8] the problem of heat and mass transfer is considered taking into account the deepening of the evaporation zone during drying. However, in the formulation of the

\footnotetext{
* Corresponding author: ivanova_tatka@mail.ru
} 
problem, the authors of Ref. 8 neglected the temperature gradients in the moist zone. It was also believed that the heat supplied by convection is completely absorbed by the evaporation of water. In [9], a system of differential equations is proposed that describes the processes of heat and moisture transfer in capillary-porous materials. But the statement [9] did not take into account the influence of structural factors of wood on the characteristics of its drying.

The temperature non-equilibrium at the boundary of the front of intensive evaporation of water in a porous medium is taken into account in [10]. To solve the problem of motion of the evaporation front, the method of accumulating the time step was used. This approach allows us to take into account a sufficiently large group of factors that significantly influence the dynamics of drying, however, this method is possible only in the case of drying objects having the properties of axial symmetry (for example, a ball).

In this paper, we consider the processes of heat and mass transfer in the convective drying of a moist porous medium in a two-dimensional formulation. The purpose of this work is mathematical modeling of drying processes of wood biomass and analysis of energy costs for carrying out the process of moisture removal.

\section{Formulation of the problem and method of solution}

The wet wood biomass is considered as an object of research. At the initial time, a sample of wood (cylindrical shape) is introduced into the high-temperature medium and heated by convection. The latter leads to the initiation of a process of evaporation of moisture. The evaporation front moves from the surface into the interior of the wood billet. As a result, a porous skeleton with high thermal resistance is formed. The solution area can be conditionally divided into two zones: dry and moist wood. Water vapor, formed during evaporation, moves from the deep layers to the surface in the radial direction. The drying of the wood ends when the moisture evaporates completely.

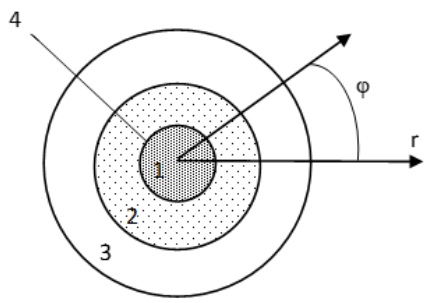

Fig. 1. Scheme of the problem solution area: 1) wet wood; 2) dry wood; 3) the region of saturation of the gaseous medium by water vapor; 4) the interface between the "wet - dry wood" system.

The drying process is described by the following system of non-stationary partial differential equations:

- the energy equation for woody biomass, taking into account the complex of heat and mass transfer processes occurring together, and the formation of a substantially heterogeneous structure, including the wet and dry parts [11]:

$$
\begin{aligned}
& \rho \frac{\partial h}{\partial \tau}+\rho_{s} C_{s}\left(U_{s} \frac{\partial T}{\partial r}+\frac{V_{s}}{r} \frac{\partial T}{\partial \varphi}\right) \Psi\left(r_{e v a}-r, \varphi_{e v a}-\varphi\right)= \\
& =\frac{1}{r} \frac{\partial}{\partial r}\left(\lambda r \frac{\partial T}{\partial r}\right)+\frac{1}{r^{2}} \frac{\partial}{\partial \varphi}\left(\lambda \frac{\partial T}{\partial \varphi}\right)-\frac{W_{e v a} Q_{e v a} \delta\left(r_{\text {eva }}, \varphi_{\text {eva }}\right)}{\Delta},
\end{aligned}
$$

where $\rho$-density of wood, $\mathrm{kg} / \mathrm{m}^{3} ; h$ - enthalpy, $\mathrm{J} / \mathrm{kg} ; \tau$ - time, $\mathrm{s} ; \rho_{\mathrm{s}}$ - density of water vapor, $\mathrm{kg} / \mathrm{m}^{3} ; C_{s}$ - heat capacity of steam, $\mathrm{J} /(\mathrm{kg} \cdot \mathrm{K}) ; U_{s}$ - speed of steam in the radial direction, 
$\mathrm{m} / \mathrm{s} ; T$ - is the temperature at the boundary of evaporation, $\mathrm{K} ; r$ - radial coordinate, $\mathrm{m} ; V_{s}$ speed of steam in the tangential direction, $\mathrm{m} / \mathrm{s} ; \varphi-$ is the azimuthal coordinate; $\psi-$ is the Heaviside function; $r_{\text {eva }}$-is the coordinate of the evaporation front, $m ; \lambda$ - thermal conductivity of wood, $\mathrm{W} /(\mathrm{m} \cdot \mathrm{K}) ; W_{\text {eva }}-$ mass evaporation rate, $\mathrm{kg} /\left(\mathrm{m}^{2} \cdot \mathrm{s}\right) ; Q_{\text {eva }}-$ is the heat of vaporization, Дж/кг; $\delta$ - is the Dirac function; $\Delta-$ is the transformation parameter of the evaporation front;

- energy equation for the gas layer around the wood [12]]:

$$
\rho \frac{\partial h}{\partial \tau}+\rho\left[U \frac{\partial h}{\partial r}+\frac{V}{r} \frac{\partial h}{\partial \varphi}\right]=\frac{\lambda}{r} \frac{\partial}{\partial r}\left(r \frac{\partial T}{\partial r}\right)+\frac{\lambda}{r^{2}} \frac{\partial^{2} T}{\partial \varphi^{2}}
$$

- the equation of piezoelectric conductivity $[13,14]$ :

$$
\frac{\partial \Pi P}{\partial \tau}=\frac{1}{r} \frac{\partial}{\partial r}\left(r \frac{K_{p}}{\beta \gamma} \frac{\partial P}{\partial r}\right)+\frac{1}{r^{2}} \frac{\partial}{\partial \varphi}\left(\frac{K_{p}}{\beta \cdot \gamma} \frac{\partial P}{\partial \varphi}\right)+\sum J_{i} \Psi\left(r_{\text {eva }}-r, \varphi_{\text {eva }}-\varphi\right),
$$

where $\Pi$ - porosity; $K_{p}$ - is the coefficient of the proportionality of the medium; $P$ - is the pressure, $\mathrm{Pa} ; \gamma$ - kinematic viscosity; $\beta$ - compressibility coefficient of water vapor; $J_{i^{-}}$is the function determining the inflow or withdrawal of the mass.

- the mass evaporation rate was calculated similarly [15]:

$$
W_{\text {eva }}=V_{0} \cdot \exp \cdot\left(\frac{\left(Q_{\text {eva }} \cdot \mu \cdot\left(T-T_{f}\right)\right)}{\left(R \cdot T_{f} \cdot T\right)}\right),
$$

where $W_{0}=2.55 \cdot 10^{-5}$ - evaporation rate at freezing point $\left(\mathrm{kg} / \mathrm{m}^{2} \mathrm{~s}\right) ; \mu=0.018--$ molar mass of water $(\mathrm{kg} / \mathrm{mol}) ; T_{f}=273$ - freezing point of water $(\mathrm{K}) ; R=8.314$ - is the universal gas constant $(\mathrm{J} / \mathrm{mol} \cdot \mathrm{K})$.

The coordinate of the evaporation boundary was found from the expression:

$$
r_{\text {eva }}=r_{0}-\int_{0}^{\tau} V d \tau,
$$

where $V=\frac{W}{\rho w}$, linear velocity of evaporation front advance, $\mathrm{m} / \mathrm{s}$.

Boundary conditions:

$$
\begin{gathered}
t=0,0<r<r_{w}, T(r, \varphi, 0)=T_{0}, r_{w}<r<r_{g}, T(r, \varphi, 0)=T_{g} ; \\
t=0,0<r<r_{w}, P(r, \varphi, 0)=P_{0}=101325 P a
\end{gathered}
$$

Border conditions:

$$
\begin{gathered}
0<\varphi<\pi, \quad \mathrm{r}=0, \quad \frac{\partial T}{\partial r}=0, \quad \frac{\partial P}{\partial r}=0 ; \\
0<\varphi<\pi, \quad \mathrm{r}=r_{d}, \quad P(\varphi, r)=P_{0} ; \\
\lambda_{d} \frac{\partial T_{1}}{\partial \varphi}=\lambda_{w} \frac{\partial T_{2}}{\partial r}, \quad r=r_{d} ; \\
T_{1}\left(r_{d}, \tau\right)=T_{2}\left(r_{d}, \tau\right) ;
\end{gathered}
$$




$$
\begin{aligned}
& r=r_{g}, \quad \frac{\partial^{2} T}{\partial r^{2}}=0, \quad 0<\varphi<\pi \\
& \varphi=0, \quad \varphi=\pi, \quad 0<r<r_{g}, \quad \frac{\partial T}{\partial \varphi}=0 .
\end{aligned}
$$

Initial data:

The ambient temperature is $T_{e}=373 \mathrm{~K}$; Specific heat of vaporization $Q_{e v a}=2.5 \cdot 10^{6} \mathrm{~J} /$ $\mathrm{kg}$; a sample of wood with radius $\mathrm{Rd}=0.0035-0.035 \mathrm{~m}$; The humidity of the sample was considered in the range $\varphi=6-40 \%$.

The thermophysical characteristics of the interacting substances were taken as follows [16]: $\lambda_{\mathrm{w}}=0.598 \mathrm{~W} / \mathrm{m} \cdot \mathrm{K} ; \rho_{\mathrm{w}}=998.2 \mathrm{~kg} / \mathrm{m}^{3} ; C_{w}=4190 \mathrm{~J} / \mathrm{kg} \cdot \mathrm{K} ; \lambda_{\text {air }}=0.026 \mathrm{~W} / \mathrm{m} \cdot \mathrm{K}$; $\rho_{\text {air }}=1.205 \mathrm{~kg} / \mathrm{m}^{3}, C_{\text {air }}=1005 \mathrm{~J} / \mathrm{kg} \cdot \mathrm{K} ; \lambda_{\mathrm{d}}=0.4 \mathrm{~W} / \mathrm{m} \cdot \mathrm{K} ; \rho_{\mathrm{d}}=510 \mathrm{~kg} / \mathrm{m}^{3}, C_{d}=2400 \mathrm{~J}$ $/ \mathrm{kg} \cdot \mathrm{K}$.

The system of equations (1-6) is solved by the method of finite differences using the algorithm [17]. Equations (1-3) are solved using quasilinear evaporation frontification using an implicit four-point difference scheme [18]. The process of heat and mass transfer in wood biomass under drying conditions is non-linear, which is due to the joint effect of a complex of physical processes occurring under conditions of intense phase transformations. As a result of evaporation, a very small, almost infinitely thin zone with an intense heat sink is formed in the porous structure of the wood [19]. Therefore, the drying process is one of the costliest technological stages in the preparation of fuelwood.

It should be noted that the results of mathematical modeling correlate well with known (published earlier) experimental data [20]. The drying time established in experiments [20] (under conditions of natural convection) is 9600 seconds. The moisture content of the wood preform was $\varphi \approx 40 \%$, the temperatures in the drying chamber varied [20] in the range from 333 to $413 \mathrm{~K}$.

Numerical studies were carried out in a fairly wide range of changes in the main parameters and process characteristics at atmospheric pressure of the environment.

\section{Results and discussion}

Based on the results of numerical simulation, the values of the total drying time for samples of woody biomass of cylindrical shape from $0.0035 \mathrm{~m}$ to $0.035 \mathrm{~m}$ were obtained.

Fig. 2 shows the characteristic drying times of the samples, determined from the results of a numerical solution of Eqs. (1-6).

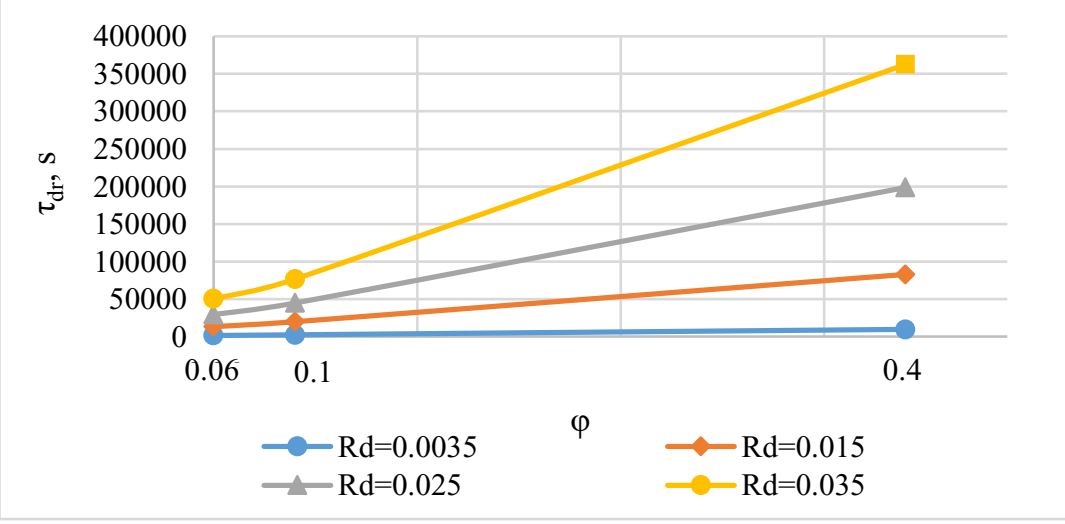

Fig. 2. Dependence of drying time of wood biomass from moisture at typical characteristic sizes of samples and ambient temperature $T e=373 \mathrm{~K}$. 
Analysis of the results showed that the wood blank of a cylindrical shape with a radius of $\mathrm{Rd}=0.035 \mathrm{~m}$ with a moisture content of $6 \%$ is completely dried in 14 hours, and at a humidity of $40 \%$, the drying time is 100 hours. Accordingly, it can be said that increasing the humidity by almost 7 times (from $6 \%$ to $40 \%$ ) results in a seven-fold lengthening of the drying period.

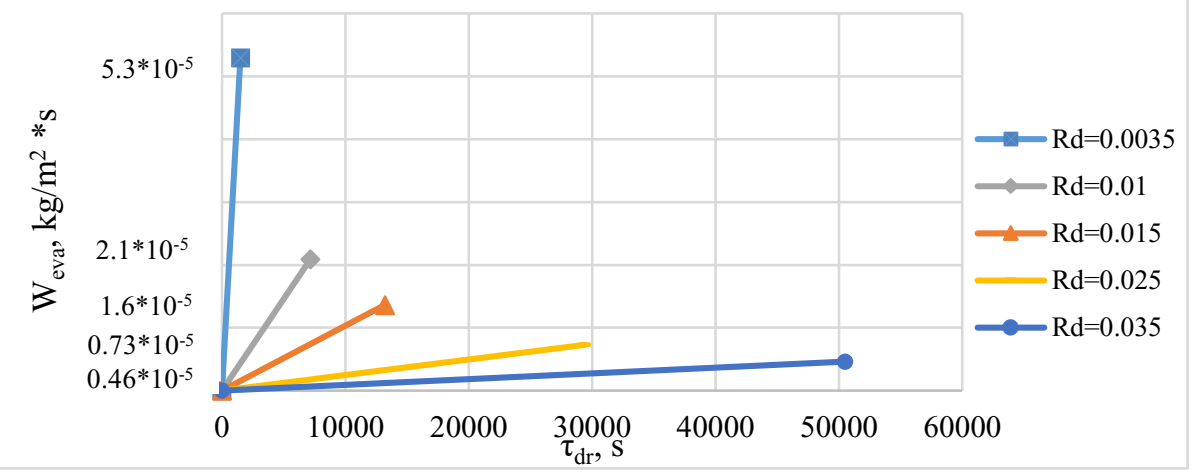

Fig. 3. Dependence of the mass evaporation rate on the drying time at ambient temperature $T e=373$ $\mathrm{K}$ and moisture content $\varphi=6 \%$ for the characteristic size of wood biomass.

The curves in Fig. 3 results allow us to conclude that from a sample of wood of cylindrical shape with a radius of $\mathrm{Rd}=0.0035 \mathrm{~m}$ with an initial humidity of $6 \%$ and an evaporation rate of $W_{\text {eva }}=5.3 \cdot 10^{-5} \mathrm{~kg} / \mathrm{m}^{2} \cdot \mathrm{s}$, all moisture is removed in 25 minutes, from the sample by radius $\mathrm{Rd}=0.035 \mathrm{~m}$ at $W_{\text {eva }}=0.46 \cdot 10^{-5} \mathrm{~kg} / \mathrm{m}^{2} \cdot \mathrm{s}$ after 14 hours. Consequently, an increase in the size of the test sample by a factor of 10 leads to a substantial increase in the drying time (by more than 30 times).

\section{Conclusion}

A new mathematical model of the processes of heat and mass transfer, proceeding together when drying wet wood, taking into account nonequilibrium parameters at the boundary of phase transitions, is developed. Based on the results of mathematical modeling, the main integral characteristics of the processes of moisture removal from wood biomass are determined. Dependences of the drying time on the initial moisture content of the wood are obtained. It is shown that an increase in humidity from $6 \%$ to $40 \%$ of a cylindrical tree billet with a radius of $0.0035 \mathrm{~m}$ results in an extension of the total drying period from 25 minutes to 2.7 hours of drying (more than 6 times).

It is established that the dimensions of the wood biomass preform have a significant effect on drying characteristics. An increase in the radius of the wood billet by a factor of 10 (from 0.0035 to $0.035 \mathrm{~m}$ ) at a humidity of $40 \%$ leads to a significant extension of the drying period (from 2.7 hours to 100 hours). Most likely, this is due to the fact that as a result of moisture removal a porous layer of dry material with high thermal resistance is formed. The latter leads to a significant delay in the heat transfer process. Also, as a result of numerical simulation, the values of the mass velocities of moisture removal from the wood billet are established for different sizes and humidity.

\section{References}

1. L.V. Zysin, N.L. Koshkin, Teploenergetika 4, 23 (1997) [in Russian]

2. L.J. R Nunes, J.C. O Matias, P. S. Catalo, Renew Sustain. Energy Rev. 40, 153 (2014)

3. M.V. Vasilevsky, E.G. Zykov, A.S. Razva, Theor. Found. Chem. Eng. 45, 304 (2011) 
4. A.S. Razva, M.V. Vasilevsky, V. Rykov, MATEC Web Conf. 23, 01035 (2015)

5. O.R. Dorniak, Forest Journal 5, 100 (2012) [in Russian]

6. R.G. Safin, R.R. Safin, R.R. Khasanshin, Forest Journal 4, 64 (2006) [in Russian]

7. N.F. Timerbaev, A.N. Grachev, R.G. Safin, Chem. Chem. Technol. 49, 103 (2006) [in Russian]

8. P.V. Akulich, N.N. Grinchik, Engineering Physics Journal 71, 225 (1998)

9. M.M. Razin, Engineering and Physics Journal 74, 290 (2001)

10. G.V. Kuznetsov, V.V. Salomatov, S.V. Syrodoy, The physics of burning and explosion 51, 409 (2015)

11. S.V. Syrodoy, G.V. Kuznetsov, N.Y. Gutareva, K.A. Bugaeva, Combust. Sci. Technol. 190, 4 (2017)

12. A.M. Grishin, S.P. Sinitsyn, I.V. Akimova, The physics of burning and explosion 6, 17 (1991)

13. Y. Chen, K. Aanjaneya, A. Atreya, Safety Journal 91, 820 (2017)

14. A. Atreyaa, P. Olszewskib, Y. Chena, H.R. Baumc, Int. J. Heat Mass Transfer 107, 319 (2017)

15. I.I. Markov, A.A. Hashchenko, O.V. Vecher, Proceedings - North Caucasian Branch of Technological Sciences of the Russian Federation 6, 48 (2002) [in Russian]

16. I.S. Grigoryev, E.Z. Meilikhov, Physical quantities (Energoatomizdat, Moscow, 1991) [in Russian]

17. S.V. Syrodoy, G.V. Kuznetsov, V.V. Salomatov, Heat power engineering 10, 1 (2015)

18. V.M. Paskonov, V.I. Polezhaev, L.A. Chudov, Numerical Modeling of Heat and Mass Transfer Processes (Science, Moscow, 1984)

19. G.V. Kuznetsov, P.A. Strizhak, Engineering and Physics Journal 87, 103 (2014)

20. E.E. Bulba, N.A. Ivanova, MATEC Web Conf. 141, 01014 (2017) DOI:10.1051/matecconf/201714101014 